\title{
Signatures of alcohol use in the structure and neurochemistry of insular cortex: a correlational study
}

\author{
Sophie Betka ${ }^{1,2,3} \mathbb{D} \cdot$ Lisa Harris $^{4} \cdot$ Charlotte Rae $^{1,5} \cdot$ Bence Palfi $^{5,6} \cdot$ Gaby Pfeifer $^{1} \cdot$ Henrique Sequeira $^{3}$. \\ Theodora Duka ${ }^{2,7} \cdot$ Hugo Critchley ${ }^{1,5}$
}

Received: 25 July 2018 / Accepted: 18 March 2019 / Published online: 22 April 2019

(C) The Author(s) 2019

\begin{abstract}
Rationale Insular cortex supports the representation of motivational feelings through the integration of interoceptive information concerning bodily physiology. Compromised insular integrity is implicated in alcohol and drug use disorders. Alcohol-associated insular dysfunction may arise through aberrant glutamatergic neurotransmission associated with selective neuronal death and atrophy.

Objective In a sample of alcohol users, we combined magnetic resonance spectroscopy (MRS) with voxel and surface-based morphometry (VBM, SBM) to test the hypothesis that the neurochemical and structural properties of the insula relate to alcohol use.

Methods Twenty-three healthy individuals were characterized by measures of alcohol use and subjective craving. Right midinsula glutamate/glutamine (Glx) and total $\mathrm{N}$-acetylaspartate/N-acetyl-aspartylglutamate (TNAA) concentrations were measured using MRS. Right insular structure was quantified using VBM and SBM parameters. We tested for predictive associations between these neuroimaging and behavioral/psychometric measures using Bayesian statistics.

Results Reduced insular Glx concentration was associated with increased alcohol compulsions and, to a lesser extent, with greater alcohol use severity. Anecdotal evidence for a negative relationship between alcohol use severity and levels of insular gyrification was also observed.

Conclusions This study is, to date, the first characterization of the neurochemical and morphological integrity of insular cortex in alcohol users. Our data seem to reveal a negative relationship between alcohol use and the neurochemical and structural integrity of the insula, a critical substrate for motivational behavior. These neurobiological characteristics might contribute to loss of control toward compulsive drinking with prolonged and excessive alcohol use.
\end{abstract}

Electronic supplementary material The online version of this article (https://doi.org/10.1007/s00213-019-05228-w) contains supplementary material, which is available to authorized users.

Sophie Betka

sophie.betka@epfl.ch

1 Trafford Centre, Brighton and Sussex Medical School, Clinical Imaging Science Centre, Brighton BN1 9RY, UK

2 Behavioural and Clinical Neuroscience, School of Psychology, University of Sussex, Brighton BN1 9QH, UK

3 University of Lille, SCALab, CNRS UMR 9193, 59045 Lille, France

4 Radiological Science, Brighton and Sussex University Hospitals NHS Trust, Brighton, UK

5 Sackler Centre for Consciousness Science, University of Sussex, Brighton, UK

6 School of Psychology, University of Sussex, Brighton, UK

7 Sussex Addiction Research and Intervention Centre (SARIC), University of Sussex, Brighton, UK
Keywords Addiction - Insula - Interoception - Magnetic resonance spectroscopy $\cdot$ Alcohol use $\cdot$ Voxel/surface-based morphometry $\cdot$ Craving

$\begin{array}{ll}\text { Abbreviations } \\ \text { AUD } & \text { Alcohol use disorders } \\ \text { AUDIT } & \text { Alcohol use disorders identification test } \\ \text { Glx } & \text { Glutamate/glutamine } \\ \text { MRS } & \text { Magnetic resonance spectroscopy } \\ \text { NMDA } & \text { N-methyl-D-aspartate } \\ \text { OCDS } & \text { Obsessive compulsive drinking scale } \\ \text { ROI } & \text { Region of interest } \\ \text { SBM } & \text { Surface-based morphometry } \\ \text { VBM } & \text { Voxel-based morphometry } \\ \text { TIV } & \text { Total intracranial volume } \\ \text { TNAA } & \text { Total N-acetylaspartate/N-acetyl-aspartylglutamate }\end{array}$

\section{Abbreviations}

AUDIT Alcohol use disorders identification test

ate/glutamine

Magnetic resonance spectroscopy

NMDA N-methyl-D-aspartate

OCDS

Region of interest

SBM Surface-based morphometry

TIV Total intracranial volume

TNAA Total $\mathrm{N}$-acetylaspartate/ $\mathrm{N}$-acetyl-aspartylglutamate 


\section{Introduction}

The insular cortex is implicated in the neurocircuitry of addiction: Interoceptive components of drug seeking (notably craving states) are proposed to originate within the insula (Craig 2002; Gray and Critchley 2007; Naqvi et al. 2007). Correspondingly, impairments in interoceptive bodily sensation are reported in drug-dependent users, including alcoholdependent users (Ates Çöl et al. 2016; Sönmez et al. 2016), methamphetamine users (Stewart et al. 2014), adolescent cannabis users (Berk et al. 2015), and people with internet gaming disorder (Zhang et al. 2016). The interconnectivity between mid-insular cortex and striatal regions is implicated in the integration and expression of hedonic experience and behavior (Chikama et al. 1997; Menon and Levitin 2005; Craig 2009, 2010). Moreover, conscious access to bodily sensations has been shown to be dependent on the right insular cortex (Craig 2002, 2009, 2010; Critchley et al. 2004). Correspondingly, damage to insular cortex can change addictive behaviors (Naqvi et al. 2014). For example, insular lesions reduce nicotine craving in smokers and attenuate the occurrence of distorted cognitive appraisals that compel betting in individuals with gambling disorder (Clark et al. 2014; Abdolahi et al. 2015). Insular volume is preferentially reduced in alcohol use disorder, in the context of more diffuse gray matter shrinkage (Yang et al. 2016), and the volume and thickness of anterior insular cortex are negatively correlated to impulsivity and compulsions in alcohol-dependent individuals (Grodin et al. 2017). Major cerebral white matter tracts are also compromised in heavy drinkers, disrupting interregional connectivity in a way that predicts an enhanced functional reactivity of insular cortex to alcohol cues. Thus, structural changes may underpin exaggerated sensitivity to cues regulating alcohol consumption (Monnig et al. 2014). Individuals with alcohol dependence also show a reduction in functional interactions between insular cortex and prefrontal regions during emotional processing, indicating a generalized dysregulation of motivational and affective processes (O'Daly et al. 2012).

In alcohol use disorders, functional brain abnormalities may be attributable to changes at the neurochemical level. Alcohol interacts with glutamatergic neurotransmission, suppressing excitatory synaptic signaling, particularly through inhibition of N-methyl-D-aspartate (NMDA) receptors (Lovinger et al. 1989). This impacts synaptic plasticity by reducing long-term potentiation (Stephens et al. 2005). In compensation, the number and sensitivity of NMDA receptors increase proportionally to the amount and frequency of alcohol intake (Trujillo and Akil 1995). A sharp reduction or cessation of alcohol consumption can induce rebound neuronal hyperexcitability, leading to excitotoxicity and neuronal death (atrophy; Tsai et al. 1995). In rats, ethanol increases glutamate concentration within striatal reward circuitry (Roberto et al. 2004; Ding et al. 2012).
In humans, it is possible to quantify neurochemicals in vivo, including glutamate, using magnetic resonance spectroscopy (MRS). However, in the literature, the impact of alcohol use on glutamate concentration seems to be mixed - potentially regionspecific. On one hand, in alcohol-dependent patients, glutamate concentration is increased within the left dorsolateral prefrontal cortex following detoxification and correlates with rated intensity of alcohol craving (Frye et al. 2016). In addition, increased glutamate levels are observed in the anterior cingulate cortex of patients in acute alcohol withdrawal (Hermann et al. 2012). Moreover, the combined concentration of glutamate and glutamine (Glx) correlates positively with compulsions to drink alcohol, measured by the Obsessive and Compulsive Drinking Scale (Anton et al. 1995), within cerebral structures widely interconnected with the insula such as the ventral striatum and anterior cingulate cortex (Bauer et al. 2013).

On the other hand, lower concentrations of glutamate and $\mathrm{N}$-acetylaspartate (NAA) within anterior cingulate cortex are observed in the early stages of stopping drinking; these concentrations seem to normalize after 5 weeks of abstinence (Mon et al. 2012). Similarly, glutamate and NAA concentrations within anterior cingulate cortex are negatively associated with recent heavy drinking in individuals with alcohol dependence (Prisciandaro et al. 2016, 2018). Finally, lower glutamate concentration within neighboring prefrontal white matter predicts loss of control and severity of alcohol dependence in heavy drinkers (Ende et al. 2013).

Together, these observations motivate the current study, in which we tested the prediction that even the "light social" drinking of alcohol impacts the neurochemical and morphological (structural) integrity of insular cortex and related regions.

\section{Present study}

Neurochemical imaging (MRS) studies of alcohol use disorders have focused on prefrontal or striatal reward-related areas. However, increasing evidence implicates insular cortex in specific aspects of drug seeking (notably hedonic information processing and craving states) (Chikama et al. 1997; Menon and Levitin 2005; Gray and Critchley 2007). Also, given the lateralization of the remapping of interoceptive signals at the insular level and its relationship with consciousness of such signals as well as the interconnectivity between midinsular cortex and striatal regions (Chikama et al. 1997; Craig 2002, 2009, 2010; Menon and Levitin 2005), we focused our investigations on the right middle insular cortex. Therefore, combining MRS with behavioral and psychometric ratings, we explored the relationship between alcohol-related measures (i.e., severity, using the Alcohol Use Disorders Identification Test), craving, and compulsion (using the Obsessive Compulsive Drinking Scale) and right middle insular cortex neurochemistry (glutamate/glutamine and TNAA metabolite concentrations) in alcohol users. 
We were also interested in associations between alcoholrelated measures, insular neurochemistry, and insular morphology (volume and surface gyrification). Indeed, glutamatergic increases can lead to excitotoxicity which might itself lead to neuronal death and potential atrophy (Lovinger et al. 1989; Tsai et al. 1995). Furthermore, majority of studies exploring alcohol-related changes in the brain structure volume have used voxel-based morphometry (VBM). However, morphology of brain structure can also be measured using a different technique such as surface-based morphometry (SBM), which measures cortical folding and is proposed to be able to capture more subtle gray matter changes (Hutton et al. 2009; Kelly et al. 2013). For example, an extensive literature is exploring the relationship between cortical folding (i.e., gyrification) and prenatal alcohol exposure in children and adolescents (De Guio et al. 2014; Kuhn et al. 2016; Hendrickson et al. 2017. 2018). However, to our knowledge, gyrification has not been quantified in adult alcohol users. Therefore, a novel aspect of this research is the combination of MRS, VBM, and SBM to clarify the neurochemical and structural integrity of insular cortex in relation to alcohol use.

First, we expected that alcohol-related psychometric measures would all be positively related. Then, based on previous reports (Yang et al. 2016), we also predicted that insular gray matter volume and cortical gyrification index would correlate negatively with these alcohol use measures. Furthermore, based on the evidence of alcohol-induced glutamatergic excitotoxicity and due to neuronal death (Lovinger et al. 1989; Tsai et al. 1995), we hypothesized that basal insular glutamate plus glutamine concentration would be lower in individuals with higher scores on alcohol use measures, as a consequence of reduced neuronal density impacting on synaptic vesicles and glutamateglutamine storage. Finally, we also explored if alcohol-related metabolites changes (e.g., glutamate-glutamine reduction) will be predicted by structural measures (e.g., gray matter reduction), suggesting an insular atrophy in alcohol users.

Here, MRS data were measured within a three-session within-subject experimental protocol that also tested for functional effects of a hormone, intranasal oxytocin, on interoceptive processes in alcohol users. There was no predicted effect of oxytocin administration on spectroscopic data (MRS glutamate/glutamine concentration). Evidence for a (nul) effect of oxytocin on metabolites was tested explicitly (see the "Materials and methods" section and Supplementary Information for more details).

\section{Material and methods}

\section{Participants}

Thirty-two male volunteers (mean age 25.1 yrs.; range 18$36 \mathrm{yrs}$ ) took part in the experiment. Participants were recruited via advertisements placed around the University of Sussex and Brighton and Sussex Medical School. All participants were healthy individuals. During the screening, participants were directly asked if they had any history or received any diagnosis of psychiatric or neurological diseases or if they were taking medication. Participants were also directly asked if they had any history or received any diagnosis of alcohol or drug use disorders. To be recruited, participants had to drink at least one unit $(8 \mathrm{~g})$ of alcohol by week. The average number of years in education was 16.9 yrs. $(\mathrm{SD}=2.6)$. All participants gave written informed consent and were compensated $£ 7$ per hour for their time. The study was reviewed and approved by the BSMS Research Governance and Ethics committee.

\section{Procedure}

The study was conducted at the Clinical Imaging Science Centre in Brighton, UK. Demographic, psychometric, and spectroscopic data were measured within a three-session within-subject experimental protocol that also tested for functional effects of a hormone, intranasal oxytocin, on interoceptive processes in alcohol users. Given the natural menstrual fluctuation of endogenous oxytocin in women, we decided to only recruit men to simplify the within-subject design procedure (e.g., women would need to be tested on the same day of their menstrual cycle). Demographic and trait psychometric data were measured during the baseline session (the first session which was not involving drug). Spectroscopic data were acquired at the end of the third (and last) session which was following the baseline session by maximum of 7 days. Each session was separated by a maximum of 2 days. Spectroscopic scan was acquired $1 \mathrm{~h}$ and $10 \mathrm{~min}$ after the drug administration. Given the short half-life of plasma oxytocin (3 to 6 min, Fabian et al. 1969), there was no predicted effect of oxytocin administration on spectroscopic data (MRS glutamate/glutamine concentration). Consistent with previous observations (Aoki et al. 2015), no evidence for an effect of oxytocin on metabolites was shown when tested for explicitly (see Supplementary Information for more details Table S1). Moreover, prior to each session, participants were breathalyzed and a urinary sample was collected to test for drug use. The urinary drug test was undertaken to confirm the absence of drug use and to exclude drug use disorder. The alcohol test was undertaken to ensure that participants abstained before the sessions. In the case of positive results, the participant would be excluded. No participants were excluded.

\section{Questionnaires}

\section{Alcohol use disorders identification test}

The alcohol use disorders identification test AUDIT (Babor et al. 2001) is a 10-item screening tool developed by the World 
Health Organization (WHO) to assess severity of alcohol use (e.g., alcohol consumption, drinking behaviors, and alcoholrelated problems). Each question is scored from 0 to 4, with higher numbers indicating a greater level of risk for having or developing an alcohol use disorder.

\section{Obsessive-compulsive drinking scale}

The Obsessive Compulsive Drinking Scale OCDS (Anton et al. 1995) is a 14-item scale measuring the obsessive and compulsive aspects of craving (e.g., drinking-related thoughts, urges to drink, and the ability to resist those thoughts and urges). The scores of "obsessions" and "compulsive drinking" subscales were calculated using published methodology (Anton et al. 1995).

\section{Magnetic resonance imaging and spectroscopy data acquisition}

Magnetic resonance imaging (MRI) and ${ }^{1} \mathrm{H}-\mathrm{MRS}$ were performed using a 1.5 Tesla Siemens Magnetom Avanto MRI scanner with an enhanced 32-channel phased-array head coil, tuned to $63.6 \mathrm{MHz}$. A whole-brain, high-resolution $\mathrm{T}_{1^{-}}$ weighted $3 \mathrm{D}$ structural image was obtained using a magnetization-prepared gradient-echo sequence, consisting of 192 contiguous axial slices $(\mathrm{TR}=2730 \mathrm{~ms}$, Echo Time $(\mathrm{TE})=3.57 \mathrm{~ms}$, flip angle $=7^{\circ}$, matrix $=256 \times 256$, field of view $(\mathrm{FoV})=256 \times 256 \mathrm{~mm}, 1.0 \mathrm{~mm}$ isotropic voxel size, GRAPPA acceleration factor $=2$; acquisition time $=5 \mathrm{~min}$ $58 \mathrm{~s}$ ). The T1-weighted image was used as an anatomical reference for each participant.

Using these images, a single ${ }^{1} \mathrm{H}-\mathrm{MRS}$ voxel was positioned in the right mid-insula of each participant (Fig. 1). A pointresolved spectroscopy sequence $(\mathrm{TR}=2 \mathrm{~s}, \mathrm{TE}=40 \mathrm{~ms}$, voxel size $=10 \times 15 \times 25 \mathrm{~mm}$, averages $=128$, flip angle $=90^{\circ}$; acquisition time $=4 \min 24 \mathrm{~s}$ ) was collected using a short TE of
$40 \mathrm{~ms}$ to minimize $\mathrm{T} 2$ losses while removing interfering macromolecular resonances (Jang et al. 2005). A shim box of the size of the voxel was used and manual shimming was performed to minimize linewidth. An unsuppressed water sequence for use as a concentration reference was collected with four averages and other identical parameters.

\section{MRI and MRS data analyses}

The $T_{1}$-weighted structural MR images were segmented into gray matter (GM), white matter (WM), and cerebrospinal liquid (CSF) using Statistical Parametric Mapping 12 (SPM12; Wellcome Department of Imaging Neuroscience, University College London, UK). To calculate the tissue content of the MRS spectroscopic voxel, a binary mask of the region in each participant was created and registered to the segmented $T_{1}$ weighted structural images. The proportion of each tissue type (tissue fractions) was calculated for the spectroscopic voxel (i.e., volume of interest) by summing the "structural" voxels of each tissue type and dividing by the total number of "structural" voxels within the volume of interest (see Supplementary Information Table S2 for details on voxel tissue content). Metabolite concentrations were then calculated and corrected (partial volume, $\mathrm{T}_{1}$, and $\mathrm{T}_{2}$ relaxation) using Gasparovic's methods (Gasparovic et al. 2006).

Raw time-domain ${ }^{1} \mathrm{H}-\mathrm{MRS}$ data in the spectral dimension were analyzed using TARQUIN Version 4.3.5 (Wilson et al. 2011) using the unsuppressed water scan as concentration reference. The quality of the model fit was verified manually. After visual inspection, any spectrum presenting one of the following characteristics was rejected from the analyses: (1) presence of obvious spectral artifacts; (2) large baseline distortion; (3) a Cramer-Rao lower bound (CRLB) of the fit to the peak of interest greater than $20 \%$. Based on these criteria, spectra of seven participants were discarded (four participants for large baseline distortion and CRLB $>20 \%$; two

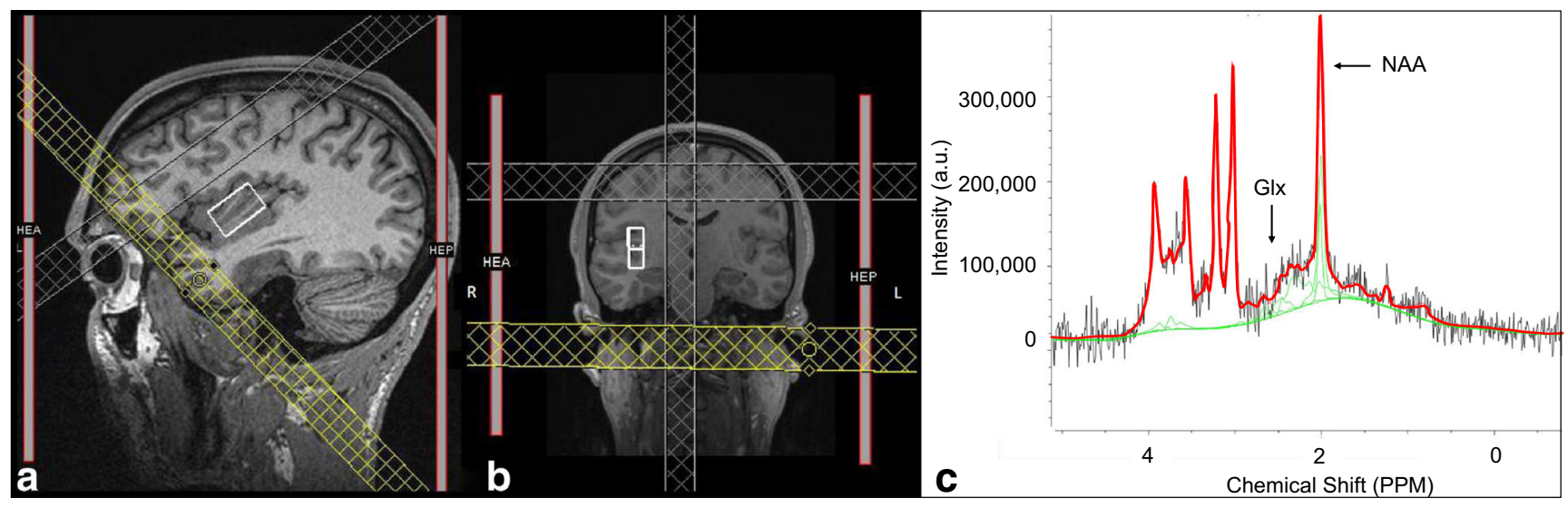

Fig. $110 \times 15 \times 25 \mathrm{~mm}$ MRS voxel placement screenshot and fitted spectrum for one participant. a Sagittal and b coronal views of MRS voxel placement in the right mid-insula, and $\mathbf{c}$ example of fitted spectrum (Glx: glutamate and glutamine; NAA: N-acetylaspartate) 
participants for presence of obvious spectral artifacts; and one for large baseline distortion, presence of obvious spectral artifacts, and CRLB > 20\%).

Metabolite concentrations in molality units of $\mathrm{mmol} / \mathrm{kg}$ of tissue water were computed for total glutamate plus glutamine (Glx) and total N-acetylaspartate plus N-acetylaspartylglutamate (TNAA). Despite technical and methodological advances, the separation of glutamate from glutamine spectral peaks is constrained by homogeneity of the magnetic field at 1.5 Tesla. Moreover, we computed the correlation coefficient $\mathrm{C}$ between derived $\mathrm{N}$-acetylaspartate and $\mathrm{N}$ acetyl-aspartylglutamate $(|\mathrm{C}|=0.756)$. Since the absolute value of the correlation coefficient between the two metabolites $|\mathrm{C}|$ was high (>0.5), then the two metabolites cannot be considered sufficiently uncorrelated to permit separation (Near 2014). Thus, the sum of the two metabolite concentrations (TNAA) was reported.

\section{Voxel and surface-based morphometry}

Voxel and surface-based morphometry (VBM, SBM) were used to identify focal differences in brain tissue composition and structure, to account for anatomical interindividual differences (Ashburner and Friston 2000; Dahnke et al. 2013). VBM and SBM were performed using the Computational Anatomy Toolbox (CAT 12 r1165, http://dbm.neuro.unijena.de/cat/).

T1-weighted structural images were realigned to the Anterior Commissure-Posterior Commissure. Image preprocessing followed established (default) settings in accordance with details described in the manual of CAT 12 toolbox (http://www.neuro.uni-jena.de/cat12/CAT12-Manual.pdf). In brief, $\mathrm{T}_{1}$-weighted 3-D images were co-registered to $\mathrm{MNI}$ space. Tissue probability maps derived from 452 healthy adults were used in affine registration and affine regularization, referencing ICBM space template-European brains. The affine processing parameter was set as default ("rough"). Medium strength correction for inhomogeneity was applied. Images were segmented into cerebrospinal fluid (CSF), gray matter (GM), and white matter (WM) using the "Adaptive Maximum A Posterior" technique. Mean estimates of gray matter volume (prior to normalization) were extracted for our region of interest (ROI): right insular cortex. The ROI was defined referencing the Neuromorphometrics Inc. atlas provided within CAT 12 under academic subscription (http:// neuromorphometrics.com/). Gray matter volume from the anterior and posterior parts of the insular cortex was averaged to give a single composite measure of insular gray matter volume.

Global gyrification index was estimated during the tissue segmentation step (Luders et al. 2006). Local cortical gyrification index was computed within CAT12 using a high-resolution parametric mesh-based approach. This approach allows estimation of the mean curvature of the brain at different spatial scales. Large positive values (expressed in degrees) for local maxima correspond to gyri. Large negative values for local minima correspond to sulci. Values are then converted to positive values by step incorporating the absolute mean curvature. Finally, the data are smoothed using a surface-based heat kernel filter of $25 \mathrm{~mm}$ resulting in revealing higher values for areas with pronounced gyrification.

Mean estimates of gyrification index were extracted (prior to normalization) for our ROI, the right insula. For each participant, ROI was labeled using the Desikan-Killiany Atlas which is included in CAT 12 (Desikan et al. 2006). Analyses were undertaken to determine the extent to which any spectroscopic concentration might relate to a reduction in insular structure. Finally, the intracranial volume (TIV) was computed for each participant using the CAT12 toolbox.

\section{Statistical analyses}

We conducted all of our analyses with the statistical software JASP, Version 0.8.6. Data were checked for potential bivariate outliers, using the function bagplot from the package "aplpack" (Rousseeuw et al. 1999) in R Version 3.5.2. Two outliers were identified and removed from the analyses. Normality of distributions was tested, given that the obsession OCDS subscale score was not normally distributed, nonparametric correlations were run.

In this present work, we tested all our hypotheses by calculating Bayesian and traditional correlation and linear regression tests. Along with frequentist statistics, we calculated the corresponding Bayes factor $(\mathrm{BF})$ which was used as the basis of decision-making in respect of the compared hypotheses. A $\mathrm{BF}$ above 3 shows compelling evidence toward the alternative hypothesis (i.e., correlation; with $1<\mathrm{BF}<3=$ anecdotal evidence; with $3<\mathrm{BF}<10=$ moderate evidence; $\mathrm{BF}>10=$ strong evidence), whereas a BF below $1 / 3$ provides substantial evidence toward the null hypothesis (i.e., there is no correlation; with $1 / 3<\mathrm{BF}<1=$ anecdotal evidence; with $1 / 10<\mathrm{BF}$ $<1 / 3=$ moderate evidence; $\mathrm{BF}<1 / 10=$ strong evidence). Thus, a BF between 3 and 1/3 implies there is not enough evidence in either direction to make a firm conclusion (Jeffreys 1961; Lee and Wagenmakers 2013). Contrary to traditional statistical methods, BFs do not need to be corrected for multiple comparisons, when all evidence relevant to the theory under assay is taken into account (Gelman et al. 2012; Dienes 2016). For each test, we also reported effect size (Cohen's f^2; Cohen 1988).

In line with the prediction that all alcohol-related psychometric measures are positively related, we applied one-tailed non-parametric correlations (Kendall's tau correlation coefficient; Kendall 1938) to test the putative positive relationship between the psychometric measures. Bayes factors $\left(\mathrm{BF}_{+\mathrm{o}}\right)$ were computed: as indicated by the subscript, the null 
hypothesis specified the absence of any association between the measures whereas the alternative hypothesis specified that the correlation is positive. To model the prior distribution of the alternative hypothesis, we chose the default prior of Kendall's $\tau$, which is a non-uniform distribution on $\tau$ produced from a uniform distribution on Pearson's $\rho$ by parametric yoking (van Doorn et al. 2018). The applied prior distribution of the alternative hypothesis assumes that high effect sizes are slightly less likely to be found as low effect sizes.

As we hypothesized that insular glutamate plus glutamine concentration would be lower in individuals with higher scores on alcohol use measures; one-tailed non-parametric correlations were again used to test the negative relationship between psychometric measures and metabolite concentrations. Bayes factors were computed $\left(\mathrm{BF}_{-}\right)$: as indicated by the subscript, the null hypothesis specified the absence of correlation whereas the alternative hypothesis specified that the correlation is negative. Again, we employed the default prior distribution of Kendall's $\tau$ for the analyses (van Doorn et al. 2018). We did not correct for TIV or age as metabolite concentrations were already corrected for the VOI tissue content.

We performed Bayesian regression analyses including model selection and test of regression slopes against zero. We applied the default settings of JASP to define the prior distributions of the compared models (Rouder and Morey 2012). Linear regressions, controlling for TIV, and age, were used to test relationships between insular (ROI) volumetric/ surface parameters, metabolite concentrations, and psychometric measures. The outcome and age as well as TIV, as control variables were entered in the null model. The outcome, age, and TIV, as control variables, and the predictor of interest were entered in the main model. For each model tested, we reported the $\mathrm{BF}$ testing for the comparison between the null and the main model. The null hypothesis was characterized by the absence of an effect of the predictor of interest on the outcome. The alternative hypothesis was characterized by the presence of an effect of the predictor of interest on the outcome. In addition to the BF, we reported effect size measures of the main model, such as R-squared and Cohen's $f^{\wedge} 2$ (Cohen 1988). Moreover, we reported the $p$ value of the model comparison procedure as well as the $p$ value of the slope of interest (i.e., whether the effect of psychometric measure on the outcome is different from zero).

\section{Results}

\section{Sample description}

Twenty-three participants were included in the analyses. All means, standard deviations, and correlations with psychometric measures were computed (Table 1). Twenty-two percent of the sample $(n=5)$ scored between 0 and 7 on the AUDIT, suggesting absence or low level of alcohol-related problems. Thirty-nine percent of the sample $(n=9)$ scored between 8 and 15 , suggesting a medium level of alcohol-related problems. Finally, $39 \%$ of the sample $(n=9)$ scored above or equal to 16 on the AUDIT, suggesting a high level of alcohol-related problems.

Strong evidence for the alternative hypothesis was observed by multiple tests: AUDIT scores were positively correlated with alcohol compulsion (compulsion subscale of the OCDS; $\left.\tau=-0.551, \mathrm{BF}_{+\mathrm{o}}=326.7, p<0.001\right)$ and alcohol obsession (obsession subscale of the OCDS; $\tau=-0.459, \mathrm{BF}_{+}$ $\mathrm{o}=46.36, p=0.003$, see Table 1 ).

\section{Voxel and surface-based morphometry-ROI analyses of right insula}

We computed linear regression to predict structural parameters based on alcohol-related measures, while controlling for TIV and age. Means and standard deviations of gray matter volume and gyrification index, as well as, regression linear model's Rsquared, Bayes factor, Cohen's $f^{\wedge} 2$, as well as uncorrected $p$ values of the model and of the slope were computed (Table 2).

Results of the linear regression predicting gyrification index from AUDIT scores, controlling for TIV, and age, showed anecdotal evidence for the alternative hypothesis: decreased gyrification index within the right insula were predicted by alcohol use severity (AUDIT) scores (Fig. 2: Model $R^{2}=$ $0.463, \mathrm{BF}=1.922$, Cohen's $f^{2}=0.225 ; p=0.007$; effect of AUDIT: $\beta=-0.371, \mathrm{SE}=0.027, t=-2.07, p=0.052$ ). The other five tests investigating the relationship between gray matter volume and psychometric measures as well as between gyrification and other psychometric measures showed anecdotal evidence for the null hypothesis (see Table 2).

\section{Insular magnetic resonance spectroscopy}

All means, standard deviations, and correlations between metabolites and psychometric measures were computed (Table 3). Substantial evidence for the alternative hypothesis was observed: Glx concentration correlated negatively with the compulsion subscale of OCDS (Fig. 3.A: $\tau=-0.383$, $\mathrm{BF}_{-0}=11.916, p=0.006$ ).

Anecdotal evidence for the alternative hypothesis was observed for the negative correlation between Glx concentration with AUDIT score (Fig. 3.B: $\tau=-0.271, \mathrm{BF}_{-0}=2.453, p=$ $0.038)$ and with the obsession subscale of OCDS $(\tau=-0.270$, $\left.\mathrm{BF}_{-0}=2.408, p=0.048\right)$. We also observed substantial evidence for the absence of a correlation between TNAA concentration and the two subscales of the OCDS (compulsion: $\tau=-0.057, \mathrm{BF}_{-0}=0.141, p=0.645$; obsession: $\tau=-0.151$, $\left.\mathrm{BF}_{-0}=0.203, p=0.824\right)$. The test probing the relationship 
Table 1 Means, standard deviations, one-tailed non-parametric coefficient correlations, Bayes Factors, and uncorrected $p$ values for psychometric measures. For all tests, the alternative hypothesis specifies that the correlation is positive $(\mathrm{BF}+0)$

\begin{tabular}{|c|c|c|c|c|}
\hline & & OCDS compulsion subscale & OCDS obsession subscale & AUDIT \\
\hline \multirow[t]{3}{*}{ OCDS obsession subscale } & Kendall's tau & 0.401 & - & \\
\hline & $\mathrm{BF}_{+0}$ & 16.00 & - & \\
\hline & $p$ value & 0.008 & - & \\
\hline \multirow[t]{5}{*}{ AUDIT } & Kendall's tau & 0.551 & 0.459 & - \\
\hline & $\mathrm{BF}_{+0}$ & 326.70 & 46.36 & - \\
\hline & $p$ value & $<0.001$ & 0.003 & - \\
\hline & Mean & 8.04 & 3.04 & 13.65 \\
\hline & SD & 4.40 & 2.72 & 7.58 \\
\hline
\end{tabular}

Support for substantial and anecdotal evidence as well as $p$ value $<0.05$ are represented in italics

between TNAA metabolite concentration and AUDIT measures showed anecdotal evidence toward the null hypothesis (see Table 3).

\section{Relationship between MRS and SBM/VBM data}

We tested if we could predict the Glx concentration from the gyrification index and gray matter volume while controlling for TIV and age. We observed anecdotal evidence for the alternative hypothesis $(3>\mathrm{BF}>1)$. However, more data are needed to confirm that Glx concentration relates positively to the insular gyrification index $\left(R^{2}=0.112, \mathrm{BF}=1.176\right.$, Cohen's $f^{2}=0.12, p=0.511$, effect of gyrification $\beta=0.39$, $\mathrm{SE}=0.4, t=1.83, p=0.156)$. Anecdotal evidence toward the null hypothesis was observed $(1 / 3<\mathrm{BF}<1)$ for a nonrelationship between Glx concentration and insular gray matter volume $\left(R^{2}=0.040, \mathrm{BF}=0.712\right.$, Cohen's $f^{2}=0.03$, $p=0.852$, effect of gray matter volume $\beta=-0.25, \mathrm{SE}=$ $1.823, t=0.773, p=0.449)$. However, as indicated by the Bayes factor, more data are needed to confirm or refute this finding.

\section{Discussion}

In the present study, we tested the relationship between alcohol-related psychometric measures (alcohol use severity, alcohol compulsion), insular neurochemical (glutamate-glutamine), and structural (volume and surface gyrification) integrity by combining MRS, VBM, and SBM. First, we observed that alcohol compulsion was associated with a reliable reduction in insular glutamate-glutamine concentration. With less
Table 2 Means and standard deviations of insular gray matter volume and gyrification index, as well as, regression linear model's R-squared, Bayes factor, effect size, and uncorrected $p$ value of the model and of the slope. For all tests, the null hypothesis was characterized by the absence of an effect of the predictor of interest on the outcome

\begin{tabular}{llll}
\hline & & Gray matter volume & Gyrification index \\
\hline AUDIT & $R^{2}$ & 0.533 & 0.463 \\
& BF & 0.452 & 1.922 \\
& Cohen's $f^{2}$ & 0.034 & 0.225 \\
& Model $p$ value & 0.002 & 0.007 \\
OCDS compulsion subscale & Slope $p$ value & 0.430 & 0.052 \\
& $R^{2}$ & 0.530 & 0.349 \\
& BF & 0.430 & 0.480 \\
& Cohen's $f^{2}$ & 0.028 & 0.011 \\
OCDS obsession subscale & Model $p$ value & 0.002 & 0.039 \\
& Slope $p$ value & 0.480 & 0.651 \\
& $\mathrm{R}^{2}$ & 0.525 & 0.386 \\
& BF & 0.398 & 0.726 \\
& Cohen's $f^{2}$ & 0.017 & 0.072 \\
& Model $p$ value & 0.002 & 0.023 \\
& Slope $p$ value & 0.576 & 0.257 \\
& Mean & 3.78 & 26.04 \\
& SD & 0.31 & 1.12 \\
\hline
\end{tabular}

Support for substantial and anecdotal evidence as well as $p$ value $<0.05$ are represented in italics 


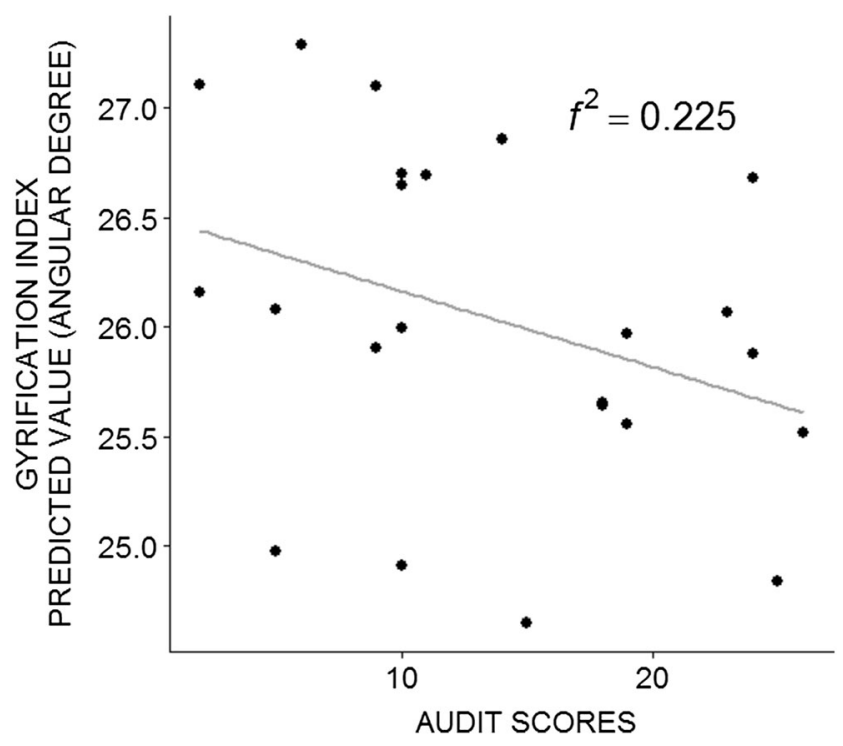

Fig. 2 Regression between predicted surface parameters and psychometric measures. Negative relationship between right insula gyrification index and AUDIT scores (controlled for age and intracranial volume). Data were checked to exclude two outliers

confidence, we observed that alcohol use severity was associated with reduced insular glutamate-glutamine concentration. Next, we found that alcohol use severity was associated with a reduction in insular gyrification. Despite their suprathreshold significance, Bayes factors for these effects indicate the need for more substantive validation.

Our initial finding was that lower combined glutamateglutamine (Glx) concentration within the mid-insular cortex reflects an increased experience of alcohol-related compulsions; metabolites concentration reduction might underpin the compulsive facet of alcohol use. While we acknowledge the limitations of dissecting the Glx peak of nuclear magnetic resonance spectra acquired at 1.5 Tesla into glutamate and glutamine, in light of previous literature, we postulate that this effect might be explained by a meaningful association between glutamate concentration and alcohol use-related measures. The meaning of a chronic elevation/reduction of glutamate-glutamine or glutamate concentration currently remains unknown, one can nevertheless infer that something has changed in the glutamatergic system. Our data, and this postulation, extend similar published observations. Indeed, in heavy drinkers, a decrease in glutamate within frontal white matter tracts (connecting insular and cingulate cortices) predicts a subjective loss of control and shift to alcohol dependency (Ende et al. 2013). Perturbed integrity of these white matter tracts may also account for decrements in functional connectivity between insula and prefrontal/cingulate cortices, which can compromise motivational regulation in people with alcohol use disorders (O'Daly et al. 2012). Our findings are also in line with the obsessive-compulsive disorder $(\mathrm{OCD})$ research literature. An MRS study noted decreased glutamate concentration in right thalamus of patients suffering from OCD: here, glutamate concentration negatively correlates with the patients' compulsion scores (Zhu et al. 2015). While the latter study did not measure glutamate within insular cortex, nevertheless insular cortices are reciprocally connected to thalami (Mufson and Mesulam 1984; Craig 2002). Interestingly, evidence suggests that repetitive Transcranial Magnetic Stimulation (rTMS) increases glutamatergic neurotransmission (Michael et al. 2003; Yue et al. 2009; Croarkin et al. 2016) and can enhance neurogenesis in animals (Ueyama et al. 2011; Zhang et al. 2014). Such rTMS can also engender increases in cortical thickness in depressed patients (Boes et al. 2018). One could postulate that neurostimulation targeting insular cortices might attenuate alcohol compulsions, via regulation of insular glutamatergic neurotransmission and the promotion of neurotrophic insular gray matter volume recovery.

An interesting finding indicated, albeit with a need for confirmation, was that lower combined glutamate-glutamine (Glx) concentration within the mid-insular cortex reflects the severity of alcohol use. Alcohol intake and acute alcohol withdrawal are typically associated with an increased glutamatergic neurotransmission and potential excitotoxity leading to neuronal death (Lovinger et al. 1989; Tsai et al. 1995; Hwa et al. 2017). However, glutamate concentration also may depend upon individual differences in drinking patterns (Ding et al. 2012), including the consequences of binge drinking episodes. Thus, our findings may reflect the impact of recent heavy drinking within a subset of our participants. Indeed, the number of heavy drinking

Table 3 Means, standard deviations, one-tailed non-parametric coefficient correlations, Bayes Factors, and uncorrected $p$ values for psychometric measures. For all tests, the alternative hypothesis specifies that the correlation is negative $\left(\mathrm{BF}_{-0}\right)$

\begin{tabular}{|c|c|c|c|c|c|c|}
\hline & & OCDS compulsion subscale & OCDS obsession subscale & AUDIT & Mean & $\mathrm{SD}$ \\
\hline \multirow[t]{3}{*}{ Glutamate + glutamine (Glx) } & Kendall's tau & -0.383 & -0.270 & -0.271 & 11.17 & 1.76 \\
\hline & $\mathrm{BF}_{-0}$ & 11.916 & 2.408 & 2.453 & & \\
\hline & $p$ value & 0.006 & 0.048 & 0.038 & & \\
\hline \multirow[t]{3}{*}{ TNAA } & Kendall's tau & 0.057 & 0.151 & -0.142 & 5.62 & 0.51 \\
\hline & $\mathrm{BF}_{-0}$ & 0.141 & 0.203 & 0.674 & & \\
\hline & $p$ value & 0.645 & 0.824 & 0.176 & & \\
\hline
\end{tabular}

Support for substantial and anecdotal evidence as well as $p$ value $<0.05$ are represented in italics 
Fig. 3 Non-parametric correlations between metabolites concentrations and psychometric measures. a Negative correlation between insular Glx concentration and compulsion subscale of the OCDS, and $\mathbf{b}$ negative correlation between insular Glx concentration and alcohol use severity indexed by AUDIT scores. Data were checked for potential outliers a

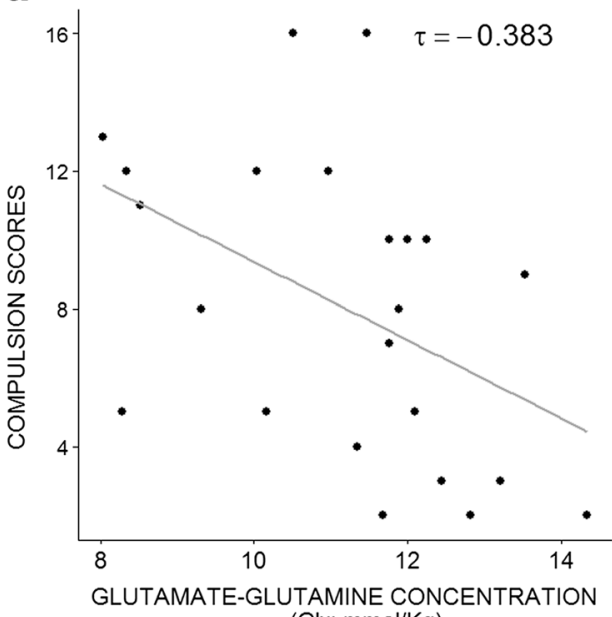

$(\mathrm{Glx} ; \mathrm{mmol} / \mathrm{Kg})$ b

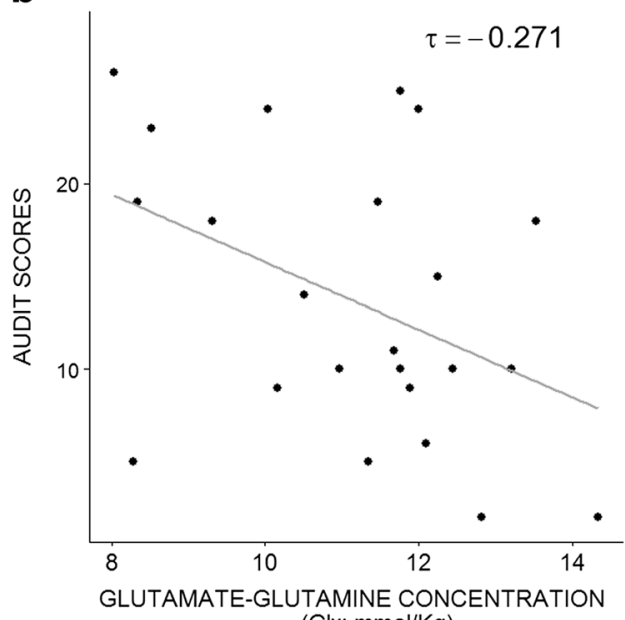

$(\mathrm{Glx} ; \mathrm{mmol} / \mathrm{Kg})$ episodes over a fortnight correlates with decreased glutamate concentration within the anterior cingulate cortex (Prisciandaro et al. 2016, 2018; Cheng et al. 2018).

There is converging evidence for insular cortex dysfunction in drug craving and addiction (Naqvi and Bechara 2010; May et al. 2013; Migliorini et al. 2013; Dinur-Klein et al. 2014; Berk et al. 2015; Senatorov et al. 2015). However, the majority of neurochemical studies of alcohol use focus on the disruptive effect of alcohol within the ventral striatum/nucleus accumbens, where mesolimbic dopamine activity signals unpredicted reward. Despite the role of insula in normal and dysfunctional motivational experience, there is a paucity of studies that specifically test the neurochemical integrity of the insular cortex in relation to alcohol use. One study reported no neurochemical differences within insular cortex of young alcohol-dependent patients, yet an increased glutamate-to-creatine ratio within the anterior cingulate cortex (Lee et al. 2007). Another study examining how glutamate and glutamine levels relate to pain processing also measured alcohol consumption in social drinkers, but again did not find any significant associations (Zunhammer et al. 2016). Of technical importance, the majority of the previously mentioned MRS studies used creatine concentration as an external reference for glutamate concentration. However, this is likely to be problematic as the stability of creatine concentration in alcohol use disorders and recreational use is questionable (Mon et al. 2012; Tunc-Skarka et al. 2015).

Notwithstanding, our findings may also indicate a potential perturbation of the glutamate-glutamine metabolic cycle associated with alcohol use, as already suggested in alcohol-dependent patients (Thoma et al. 2011). This disruption is, putatively, a neurobiological risk factor for alcohol use disorders, rather than an incidental consequence of alcohol drinking. In this context, abnormalities in the glutamate/glutamine metabolic cycle within anterior cingulate cortex correlate with higher impulsivity in youths with a family history of alcoholism (Cohen-Gilbert et al. 2015). However, within the present study, given the low magnetic field, glutamate and glutamine concentrations are not confidently differentiable in our study. Nevertheless, our findings indicate the potential presence of alcohol-related glutamate/glutamine reduction within the right insula, which may underlie a pathogenetic vicious cycle of alcohol craving and further drinking.

Our second main findings indicate anecdotal evidence toward a negative relationship between the gyrification of the right insular cortex and alcohol drinking severity, confirming a priori predictions. To our knowledge, the present study is the first study to quantify such a relationship between insular gyrification with respect to alcohol use. Insular atrophy is commonly reported in alcohol-dependent individuals (Yang et al. 2016) and might account within our sample for part of the relationship between increased alcohol use severity and a reduction in gyrification (through gray matter loss). Notwithstanding, given the established relationship between prenatal alcohol exposure, reduced cortical folding, and higher risk to develop alcohol use disorders later in life (De Guio et al. 2014; Kuhn et al. 2016; Hendrickson et al. 2017, 2018); one cannot exclude the fact that our observations might reflect a predisposition to, rather than an effect of, alcohol consumption. Longitudinal studies are needed to disentangle this point. In addition, we observed no specific relationship between alcohol-related measures and overall gray matter volume of the insular cortex. This finding suggests that surface-based morphometry parameters might be a more sensitive measure of early alcohol-associated decline in structural gray matter organization than voxel-based morphometry estimates, as already suggested by two previous studies (Hutton et al. 2009; Kelly et al. 2013).

In our study, we also tested whether insular metabolite concentration was predicted by insular gyrification, suggesting subtle atrophy. Indeed, glutamatergic increases can lead to excitotoxicity and neuronal death (Lovinger et al. 1989; Tsai et al. 1995). It is, therefore, possible that the observed reduction in insular glutamate-glutamine concentration is a result of reduced number of cells (e.g., neural death consequent upon 
alcohol-related glutamatergic excitotoxicity), indexed here as subtle changes in insular gyrification. Unfortunately, our anecdotal evidence was insufficient to support this theory; future studies combining MRS and structural measures are needed to explore this point.

Nevertheless, our findings seem to indicate a combined disruption of right insular neurochemistry and structure, which seem to be associated with alcohol craving and alcohol use. The insular cortex processes afferent interoceptive signals, which are a likely basis to craving states (Critchley et al. 2004; Naqvi et al. 2007). Indeed, the disruption of interoceptive processes in alcohol-dependent individuals correlates positively with subjective craving ratings (Ates Çöl et al. 2016; Sönmez et al. 2016) and, more generally, impaired processing of bodily sensation is linked to insular cortex dysfunction in drug use disorders (Stewart et al. 2014). Recently, we observed effects compatible with an impairment in switching attention between interoceptive and exteroceptive signals in heavy drinkers (Betka et al. 2018). A similar impairment is documented in patients suffering from OCD (Stern et al. 2017). This is consistent with the important role that insular cortex plays in salience attribution (Seeley et al. 2007, Sridharan et al. 2008, Menon and Uddin 2010). Hence, reduced structural and neurochemical insular integrity might partly explain such impairment. However, it would be helpful to pursue neuroimaging studies to define functional neural correlates of pure visceral interoception (independently of other sources of bodily sensations, notably cutaneous touch or proprioceptive sensations of muscular effort) and the capacity to switch attention between salient interoception/ exteroception cues, both in individuals with alcohol use disorders and those with non-clinical patterns of alcohol use.

\section{Limitations}

The results of the present study should be considered in light of several constraints. First, we recognize that further information concerning aspects of alcohol consumption may have provided further mechanistic insight into pathoaetiological processes; including knowledge of starting age of alcohol intake, frequency, and precise quantity alcohol intake over different stages of the lifespan. Moreover, we did not formally elicit family histories of alcohol use disorder, which also impacts neurochemical ratio and morphological brain integrity (Cohen-Gilbert et al. 2015). A detailed alcohol consumption history for the preceding 2 weeks before neuroimaging may have excluded more acute factors underlying differences in neurochemical levels, as recent heavy drinking episodes can perturb glutamatergic neurotransmission (Prisciandaro et al. 2016, 2018). Also, although we controlled for alcohol and drug abstinence before the study, our findings might have been further enhanced by additionally controlling for tobacco smoking habits: Alcohol-dependent individuals who smoke show reduced $\mathrm{N}$-acetylaspartate cerebral concentration when compared to alcohol-dependent non-smokers (Durazzo et al. 2013). In this regard, a strict screening for drug use or drug history should be added to protocols of future studies as many substances have long-term effects on glutamate brain level. In addition, a recognized technical limitation was the magnetic strength of the MRI scanner for neurochemical discrimination using MRS. A higher field strength can enable more direct, separate quantification of GABA, glutamate, and glutamine concentrations. Also, a greater magnetic field would increase the signal/noise ratio, which would have been beneficial given the relatively small voxel size $(10 \times 15 \times 25 \mathrm{~mm})$ necessary to derive neurochemical concentrations from insula. Bayes factors indicating anecdotal evidence for a number of effects highlight the need for a larger group size. Lastly, our sample was exclusively composed of males; therefore, further studies should verify the generalization of our findings in females.

\section{Conclusion}

We quantified the neurochemical and morphological integrity of the insular cortex in alcohol users. Together, our data provide evidence for disruption of insular glutamate-glutamine concentration and a modulation of brain surface parameters by alcohol use. These changes may underpin a loss of control over alcohol and a shift toward compulsive drinking. Further (longitudinal) studies should explore the evolution of interoceptive processes in relation to the integrity of insular cortex through different developmental stages of alcohol and drug disorders.

Acknowledgements We thank Dr. Jessica Eccles, Dr. Marta Łukowska, Joely Smith, and Dr. Nick Dowell for their assistance.

Funding The present study was supported in part by Rotary Foundation (Rotary Global Grant GG1526999), the Society for the Study of Addiction, and the European Research Council (Advanced Grant CCFIB AG 234150 awarded to HDC) and the European Union's Horizon 2020 program (668863-SyBil-AA; awarded to TD and HDC). Sophie Betka is grateful to the Society for the Study of Addiction (SSA) for funding support under their $\mathrm{PhD}$ Studentship scheme and states that the opinions expressed are those of the author(s) and do not necessarily reflect the views of the SSA itself.

\section{Compliance with ethical standards}

Conflict of interest On behalf of all authors, the corresponding author states that there is no conflict of interest.

Open Access This article is distributed under the terms of the Creative Commons Attribution 4.0 International License (http:// creativecommons.org/licenses/by/4.0/), which permits unrestricted use, distribution, and reproduction in any medium, provided you give appropriate credit to the original author(s) and the source, provide a link to the Creative Commons license, and indicate if changes were made. 


\section{References}

Abdolahi A, Williams GC, Benesch CG, Wang HZ, Spitzer EM, Scott BE, Block RC, van Wijngaarden E (2015) Damage to the insula leads to decreased nicotine withdrawal during abstinence. Addiction 110(12):1994-2003

Anton RF, Moak DH, Latham P (1995) The Obsessive Compulsive Drinking Scale: a self-rated instrument for the quantification of thoughts about alcohol and drinking behavior. Alcohol Clin Exp Res 19(1):92-99

Aoki Y, Watanabe T, Abe O, Kuwabara H, Yahata N, Takano Y, Iwashiro N, Natsubori T, Takao H, Kawakubo Y, Kasai K, Yamasue H, (2015) Oxytocin's neurochemical effects in the medial prefrontal cortex underlie recovery of task-specific brain activity in autism: a randomized controlled trial Mol Psychiatry. Apr 20(4):447-53. https://doi.org/10.1038/mp.2014.74

Ashburner J, Friston KJ (2000) Voxel-based morphometry-the methods. Neuroimage 11(6 Pt 1):805-821

Ates Cöl I, Sömnez MB, Vardar ME (2016) Evaluation of interoceptive awareness in alcohol-addicted patients. Arch Neuropsychiatry 53: $17-22$

Babor T, Higgins-Biddle J, Saunders J, Monteiro M (2001) AUDIT: The Alcohol Use Disorders Identification Test, second edn. Department of Mental Health and Substance Dependence, Geneva

Bauer J, Pedersen A, Scherbaum N, Bening J, Patschke J, Kugel H, Heindel W, Arolt V, Ohrmann P (2013) Craving in alcoholdependent patients after detoxification is related to glutamatergic dysfunction in the nucleus accumbens and the anterior cingulate cortex. Neuropsychopharmacology 38(8):1401-1408. https://doi. org/10.1038/npp.2013.45

Berk L, Stewart JL, May AC, Wiers RW, Davenport PW, Paulus MP, Tapert SF (2015) Under pressure: adolescent substance users show exaggerated neural processing of aversive interoceptive stimuli. Addiction 110(12):2025-2036

Betka S, Gould Van Praag C, Paloyelis Y, Bond R, Pfeifer G, Sequeira H, Duka T, Critchley H (2018) Impact of intranasal oxytocin on interoceptive accuracy in alcohol users: an attentional mechanism? Soc Cogn Affect Neurosci. https://doi.org/10.1093/scan/nsy027

Boes AD, Uitermarkt BD, Albazron FM, Lan MJ, Liston C, PascualLeone A, Dubin MJ, Fox MD (2018) Rostral anterior cingulate cortex is a structural correlate of repetitive TMS treatment response in depression. Brain Stimul 11(3):575-581. https://doi.org/10.1016/ j.brs.2018.01.029

Cheng H, Kellar D, Lake A, Finn P, Rebec GV, Dharmadhikari S, Dydak U, Newman S (2018) Effects of alcohol cues on MRS glutamate levels in the anterior cingulate. Alcohol Alcohol 53(3):209-215

Chikama M, McFarland NR, Amaral DG, Haber SN (1997) Insular cortical projections to functional regions of the striatum correlate with cortical cytoarchitectonic organization in the primate. J Neurosci 17(24):9686-9705

Clark L, Studer B, Bruss J, Tranel D, Bechara A (2014) Damage to insula abolishes cognitive distortions during simulated gambling. PNAS 111(16):6098-6103

Cohen JE (1988) Statistical power analysis for the behavioral sciences. Lawrence Erlbaum Associates, Inc., Hillsdale

Cohen-Gilbert JE, Sneider JT, Crowley DJ, Rosso IM, Jensen JE, Silveri MM (2015) Impact of family history of alcoholism on glutamine/ glutamate ratio in anterior cingulate cortex in substance-naïve adolescents. Dev Cogn Neurosci 16:147-154

Craig AD (2002) How do you feel? Interoception: the sense of the physiological condition of the body. Nat Rev Neurosci 3(8):655-666
Craig AD (2009) How do you feel-now? The anterior insula and human awareness. Nat Rev Neurosci 10(1):59-70. https://doi.org/10.1038/ $\operatorname{nrn} 2555$

Craig AD (2010) The sentient self. Brain Struct Funct 214(5-6):563-577

Critchley HD, Wiens S, Rotshtein P, Ohman A, Dolan RJ (2004) Neural systems supporting interoceptive awareness. Nat Neurosci 7(2): 189-195

Croarkin PE, Nakonezny PA, Wall CA, Murphy LL, Sampson SM, Frye MA, Port JD (2016) Transcranial magnetic stimulation potentiates glutamatergic neurotransmission in depressed adolescents. Psychiatry Res 247:25-33

Dahnke R, Yotter RA, Gaser C (2013) Cortical thickness and central surface estimation. Neuroimage 65:336-348

De Guio F, Mangin JF, Riviere D, Perrot M, Molteno CD, Jacobson SW, Meintjes EM, Jacobson JL (2014) A study of cortical morphology in children with fetal alcohol spectrum disorders. Hum Brain Mapp 35(5):2285-2296

Desikan RS, Segonne F, Fischl B, Quinn BT, Dickerson BC, Blacker D, Buckner RL, Dale AM, Maguire RP, Hyman BT, Albert MS, Killiany RJ (2006) An automated labeling system for subdividing the human cerebral cortex on MRI scans into gyral based regions of interest. Neuroimage 31(3):968-980

Dienes Z (2016) How Bayes factors change scientific practice. J Math Psychol 72:78-89

Ding ZM, Engleman EA, Rodd ZA, McBride WJ (2012) Ethanol increases glutamate neurotransmission in the posterior ventral tegmental area of female Wistar rats. Alcohol Clin Exp Res 36(4):633-640

Dinur-Klein L, Dannon P, Hadar A, Rosenberg O, Roth Y, Kotler M, Zangen A (2014) Smoking cessation induced by deep repetitive transcranial magnetic stimulation of the prefrontal and insular cortices: a prospective, randomized controlled trial. Biol Psychiatry 76(9):742-749

Durazzo TC, Mon A, Gazdzinski S, Meyerhoff DJ (2013) Chronic cigarette smoking in alcohol dependence: associations with cortical thickness and $\mathrm{N}$-acetylaspartate levels in the extended brain reward system. Addict Biol 18(2):379-391

Ende G, Hermann D, Demirakca T, Hoerst M, Tunc-Skarka N, WeberFahr W, Wichert S, Rabinstein J, Frischknecht U, Mann K, Vollstadt-Klein S (2013) Loss of control of alcohol use and severity of alcohol dependence in non-treatment-seeking heavy drinkers are related to lower glutamate in frontal white matter. Alcohol Clin Exp Res 37(10):1643-1649

Fabian M, Forsling ML, Jones JJ, Pryor JS (1969) The clearance and antidiuretic potency of neurohypophysial hormones in man, and their plasma binding and stability. J Physiol 204(3):653-668

Frye MA, Hinton DJ, Karpyak VM, Biernacka JM, Gunderson LJ, Geske J, Feeder SE, Choi DS, Port JD (2016) Elevated glutamate levels in the left dorsolateral prefrontal cortex are associated with higher cravings for alcohol. Alcohol Clin Exp Res 40(8):1609-1616

Gasparovic C, Song T, Devier D, Bockholt HJ, Caprihan A, Mullins PG, Posse S, Jung RE, Morrison LA (2006) Use of tissue water as a concentration reference for proton spectroscopic imaging. Magn Reson Med 55(6):1219-1226

Gelman A, Hill J, Yajima M (2012) Why we (usually) don't have to worry about multiple comparisons. J Res Educ Effect:189-211. https://doi.org/10.1080/19345747.2011.618213

Gray MA, Critchley HD (2007) Interoceptive basis to craving. Neuron 54(2):183-186. https://doi.org/10.1016/j.neuron.2007.03.024

Grodin EN, Cortes CR, Spagnolo PA, Momenan R (2017) Structural deficits in salience network regions are associated with increased impulsivity and compulsivity in alcohol dependence. Drug Alcohol Depend 179:100-108

Hendrickson TJ, Mueller BA, Sowell ER, Mattson SN, Coles CD, Kable JA, Jones KL, Boys CJ, Lim KO, Riley EP, Wozniak JR (2017) Cortical gyrification is abnormal in children with prenatal alcohol exposure. Neuroimage Clin 15:391-400 
Hendrickson TJ, Mueller BA, Sowell ER, Mattson SN, Coles CD, Kable JA, Jones KL, Boys CJ, Lee S, Lim KO, Riley EP, Wozniak JR (2018) Two-year cortical trajectories are abnormal in children and adolescents with prenatal alcohol exposure. Dev Cogn Neurosci 30: $123-133$

Hermann D, Weber-Fahr W, Sartorius A, Hoerst M, Frischknecht U, Tunc-Skarka N, Perreau-Lenz S, Hansson AC, Krumm B, Kiefer F, Spanagel R, Mann K, Ende G, Sommer WH (2012) Translational magnetic resonance spectroscopy reveals excessive central glutamate levels during alcohol withdrawal in humans and rats. Biol Psychiatry 71(11):1015-1021

Hutton C, Draganski B, Ashburner J, Weiskopf N (2009) A comparison between voxel-based cortical thickness and voxel-based morphometry in normal aging. Neuroimage 48(2):371-80. https://doi.org/10. 1016/j.neuroimage.2009.06.043

Hwa L, Besheer J, Kash T (2017) Glutamate plasticity woven through the progression to alcohol use disorder: a multi-circuit perspective. F1000Res 6:298

Jang DP, Lee JM, Lee E, Park S, Kim JJ, Namkoong K, Yoon KJ, Kim IY, Kim SI (2005) Interindividual reproducibility of glutamate quantification using 1.5-T proton magnetic resonance spectroscopy. Magn Reson Med 53(3):708-712

Jeffreys H (1961) Theory of probability, 3rd edn. Oxford University Press, Oxford

Kelly PA, Viding E, Wallace GL, Schaer M, De Brito SA, Robustelli B, McCrory EJ (2013) Cortical thickness, surface area, and gyrification abnormalities in children exposed to maltreatment: neural markers of vulnerability? Biol Psychiatry 74(11):845-852

Kendall M (1938) A new measure of rank correlation. Biometrika 30:8193

Kuhn S, Witt C, Banaschewski T, Barbot A, Barker GJ, Buchel C, Conrod PJ, Flor H, Garavan H, Ittermann B, Mann K, Martinot JL, Paus T, Rietschel M, Smolka MN, Strohle A, Bruhl R, Schumann G, Heinz A, Gallinat J (2016) From mother to child: orbitofrontal cortex gyrification and changes of drinking behaviour during adolescence. Addict Biol 21(3):700-708

Lee M, Wagenmakers E-J (2013) Bayesian modeling for cognitive science: a practical course. Cambridge University Press, Cambridge

Lee E, Jang DP, Kim JJ, An SK, Park S, Kim IY, Kim SI, Yoon KJ, Namkoong K (2007) Alteration of brain metabolites in young alcoholics without structural changes. Neuroreport 18(14):1511-1514

Lovinger DM, White G, Weight FF (1989) Ethanol inhibits NMDAactivated ion current in hippocampal neurons. Science 243(4899): 1721-1724

Luders E, Thompson PM, Narr KL, Toga AW, Jancke L, Gaser C (2006) A curvature-based approach to estimate local gyrification on the cortical surface. Neuroimage 29(4):1224-1230

May AC, Stewart JL, Migliorini R, Tapert SF, Paulus MP (2013) Methamphetamine dependent individuals show attenuated brain response to pleasant interoceptive stimuli. Drug Alcohol Depend 131(3):238-246

Menon V, Levitin DJ (2005) The rewards of music listening: response and physiological connectivity of the mesolimbic system. Neuroimage 28(1):175-184

Menon V, Uddin LQ (2010) Saliency, switching, attention and control: a network model of insula function. Brain Struct Funct 214(5-6):655667

Michael N, Gosling M, Reutemann M, Kersting A, Heindel W, Arolt V, Pfleiderer B (2003) Metabolic changes after repetitive transcranial magnetic stimulation (rTMS) of the left prefrontal cortex: a shamcontrolled proton magnetic resonance spectroscopy (1H MRS) study of healthy brain. Eur J Neurosci 17(11):2462-2468

Migliorini R, Stewart JL, May AC, Tapert SF, Paulus MP (2013) What do you feel? Adolescent drug and alcohol users show altered brain response to pleasant interoceptive stimuli. Drug Alcohol Depend 133(2):661-668
Mon A, Durazzo TC, Meyerhoff DJ (2012) Glutamate, GABA, and other cortical metabolite concentrations during early abstinence from alcohol and their associations with neurocognitive changes. Drug Alcohol Depend 125(1-2):27-36

Monnig MA, Thayer RE, Caprihan A, Claus ED, Yeo RA, Calhoun VD, Hutchison KE (2014) White matter integrity is associated with alcohol cue reactivity in heavy drinkers. Brain Behav 4(2):158-170

Mufson EJ, Mesulam MM (1984) Thalamic connections of the insula in the rhesus monkey and comments on the paralimbic connectivity of the medial pulvinar nucleus. J Comp Neurol 227(1):109-120

Naqvi NH, Bechara A (2010) The insula and drug addiction: an interoceptive view of pleasure, urges, and decision-making. Brain Struct Funct 214(5-6):435-450

Naqvi NH, Rudrauf D, Damasio H, Bechara A (2007) Damage to the insula disrupts addiction to cigarette smoking. Science 315(5811): 531-534

Naqvi NH, Gaznick N, Tranel D, Bechara A (2014) The insula: a critical neural substrate for craving and drug seeking under conflict and risk. Ann N Y Acad Sci 1316:53-70

Near J (2014) Magnetic resonance spectroscopy tools for neuroscience research and emerging clinical applications. San Diego, State: California

O'Daly OG, Trick L, Scaife J, Marshall J, Ball D, Phillips ML, Williams SS, Stephens DN, Duka T (2012) Withdrawal-associated increases and decreases in functional neural connectivity associated with altered emotional regulation in alcoholism. Neuropsychopharmacology::2267-2276

Prisciandaro JJ, Schacht JP, Prescot AP, Renshaw PF, Brown TR, Anton RF (2016) Associations between recent heavy drinking and dorsal anterior cingulate $\mathrm{N}$-acetylaspartate and glutamate concentrations in non-treatment-seeking individuals with alcohol dependence. Alcohol Clin Exp Res 40(3):491-496

Prisciandaro JJ, Schacht JP, Prescot AP, Renshaw PF, Brown TR, Anton RF (2018) Brain glutamate, GABA, and glutamine levels and associations with recent drinking in treatment-naive individuals with alcohol use disorder versus light drinkers. Alcohol Clin Exp Res 43(2):221-226. https://doi.org/10.1111/acer.13931

Roberto M, Schweitzer P, Madamba SG, Stouffer DG, Parsons LH, Siggins GR (2004) Acute and chronic ethanol alter glutamatergic transmission in rat central amygdala: an in vitro and in vivo analysis. J Neurosci 24(7):1594-1603

Rouder JN, Morey RD (2012) Default Bayes factors for model selection in regression. Multivar Behav Res 47(6):877-903

Rousseeuw PJ, Ruts I, Tukey JW (1999) The bagplot: a bivariate boxplot. Am Stat 53:382-387. https://doi.org/10.1080/00031305.1999. 10474494

Seeley WW, Menon V, Schatzberg AF, Keller J, Glover GH, Kenna H, Reiss AL, Greicius MD (2007) Dissociable intrinsic connectivity networks for salience processing and executive control. J Neurosci 27(9):2349-2356

Senatorov VV, Damadzic R, Mann CL, Schwandt ML, George DT, Hommer DW, Heilig M, Momenan R (2015) Reduced anterior insula, enlarged amygdala in alcoholism and associated depleted von Economo neurons. Brain 138(Pt 1):69-79

Sönmez MB, Kahyacı Kılıc̣ E, Ateș Cöl I, Görgülü Y, Köse Çınar R (2016) Decreased interoceptive awareness in patients with substance use disorders. J Subst Use 22:60-65. https://doi.org/10.3109/ 14659891.2016.1143048

Sridharan D, Levitin DJ, Menon V (2008) A critical role for the right fronto-insular cortex in switching between central-executive and default-mode networks. Proc Natl Acad Sci U S A 105(34): 12569-12574

Stephens DN, Ripley TL, Borlikova G, Schubert M, Albrecht D, Hogarth L, Duka T (2005) Repeated ethanol exposure and withdrawal impairs human fear conditioning and depresses long-term potentiation in rat amygdala and hippocampus. Biol Psychiatry 58(5):392-400 
Stern ER, Muratore AF, Taylor SF, Abelson JL, Hof PR, Goodman WK (2017) Switching between internally and externally focused attention in obsessive-compulsive disorder: abnormal visual cortex activation and connectivity. Psychiatry Res 265:87-97

Stewart JL, May AC, Poppa T, Davenport PW, Tapert SF, Paulus MP (2014) You are the danger: attenuated insula response in methamphetamine users during aversive interoceptive decision-making. Drug Alcohol Depend 142:110-119

Thoma R, Mullins P, Ruhl D, Monnig M, Yeo RA, Caprihan A, Bogenschutz M, Lysne P, Tonigan S, Kalyanam R, Gasparovic C (2011) Perturbation of the glutamate-glutamine system in alcohol dependence and remission. Neuropsychopharmacology 36(7): 1359-1365. https://doi.org/10.1038/npp.2011.20

Trujillo KA, Akil H (1995) Excitatory amino acids and drugs of abuse: a role for N-methyl-D-aspartate receptors in drug tolerance, sensitization and physical dependence. Drug Alcohol Depend 38(2):139 154

Tsai G, Gastfriend DR, Coyle JT (1995) The glutamatergic basis of human alcoholism. Am J Psychiatry 152(3):332-340

Tunc-Skarka N, Weber-Fahr W, Ende G (2015) Recreational alcohol use induces changes in the concentrations of choline-containing compounds and total creatine in the brain: a (1)H MRS study of healthy subjects. Magma 28(5):503-510

Ueyama E, Ukai S, Ogawa A, Yamamoto M, Kawaguchi S, Ishii R, Shinosaki K (2011) Chronic repetitive transcranial magnetic stimulation increases hippocampal neurogenesis in rats. Psychiatry Clin Neurosci 65(1):77-81

van Doorn J, Ly A, Marsman M, Wagenmakers E-J (2018) Bayesian inference for Kendall's rank correlation coefficient. Am Stat 72:16. https://doi.org/10.1080/00031305.2016.1264998

Wilson M, Reynolds G, Kauppinen RA, Arvanitis TN, Peet AC (2011) A constrained least-squares approach to the automated quantitation of in vivo (1)H magnetic resonance spectroscopy data. Magn Reson Med 65(1):1-12

Yang X, Tian F, Zhang H, Zeng J, Chen T, Wang S, Jia Z, Gong Q (2016) Cortical and subcortical gray matter shrinkage in alcohol-use disorders: a voxel-based meta-analysis. Neurosci Biobehav Rev 66:92-103

Yue L, Xiao-lin H, Tao S (2009) The effects of chronic repetitive transcranial magnetic stimulation on glutamate and gamma-aminobutyric acid in rat brain. Brain Res 1260:94-99

Zhang Y, Mao RR, Chen ZF, Tian M, Tong DL, Gao ZR, Huang M, Li X, Xu X, Zhou WH, Li CY, Wang J, Xu L, Qiu Z (2014) Deep-brain magnetic stimulation promotes adult hippocampal neurogenesis and alleviates stress-related behaviors in mouse models for neuropsychiatric disorders. Mol Brain 7:11. https://doi.org/10.1186/1756-6606$7-11$

Zhang Y, Mei W, Zhang JX, Wu Q, Zhang W (2016) Decreased functional connectivity of insula-based network in young adults with internet gaming disorder. Exp Brain Res 234:2553-2560

Zhu Y, Fan Q, Han X, Zhang H, Chen J, Wang Z, Zhang Z, Tan L, Xiao Z, Tong S, Maletic-Savatic M, Li Y (2015) Decreased thalamic glutamate level in unmedicated adult obsessive-compulsive disorder patients detected by proton magnetic resonance spectroscopy. J Affect Disord 178:193-200

Zunhammer M, Schweizer LM, Witte V, Harris RE, Bingel U, SchmidtWilcke T (2016) Combined glutamate and glutamine levels in painprocessing brain regions are associated with individual pain sensitivity. Pain 157(10):2248-2256

Publisher's note Springer Nature remains neutral with regard to jurisdictional claims in published maps and institutional affiliations. 